\title{
Die deutsch-jüdische Emigration nach Argentinien (1933-1939)
}

\section{Elena Levin}

Die vorliegende Arbeit stellt einen Versuch dar, die Charakteristika jüdischer Emigranten aus Deutschland in Argentinien in den Jahren 1933 bis 1939 zu analysieren und zu ihrem Verständnis beizutragen. Sie ist das Ergebnis einer bibliographischen Studie sowie der Befragung von 41 deutschen Juden, die gegenwärtig in Buenos Aires leben. Dabei wurde besonders auf die Methodik der "oral history" zurückgegriffen, um das Phänomen des jüdischen Exilanten zu untersuchen, das - so A. Schrader - in besonderer Weise behandelt werden muß, da es nicht nur Produkt des Genozids, sondern einer über dreitausend Jahre währenden Diaspora ist. Befragt wurden 41 Personen, die die Erstellung einer Art Profil ermöglichen: Es erlaubt uns, bestimmte Verhaltensweisen zu beobachten und zu hinterfragen und einige besondere Charakteristika herauszustellen, die darauf schließen lassen, daß diese deutschen Juden als Gruppe sich von anderen ethnischen Gruppen und anderen Juden in Argentinien unterscheiden.

Der Begriff "Jude" wird hier in einem weiten Sinn gebraucht, der über rein religiöse, nationalistische oder einseitige Interpretationen hinausgeht. In diesem Text steht die Bezeichnung für jeden, der sich aus familiären oder sozialen Gründen heraus auf irgendeine Weise mit den kulturellen, religiösen, philosophischen oder historischen Traditionen des jüdischen Volkes verbunden fühlt. 41 in Deutschland geborene Personen wurden für die Befragung ausgewählt, wobei unter Deutschland das Deutsche Reich in seinen damaligen geographischen Grenzen unter Einbeziehung des Saarlandes (das nach der Volksbefragung vom 1. November 1935 erneut zu Deutschland kam) zu verstehen ist.

Bereits im 4. Jahrhundert n. Chr. gab es jüdische Gemeinden in allen Provinzen des Römischen Imperiums und somit auch in Germanien. Die Juden genossen Glaubensfreiheit, und es war ihnen gestattet, ihre Streitfälle nach jüdischem Recht zu entscheiden. Vom Militärdienst waren sie befreit. Kaiser Konstantin erlaubte in einem Dokument aus dem Jahre 321 einer jüdischen Gemeinde, sich in der Stadt Köln anzusiedeln. Fast 1600 Jahre später lebten laut einer Volkszählung aus dem Jahre 1925564.379 Juden in Deutschland. Seit dem 19. Jahrhundert hatten Juden wichtige Regierungsämter bekleidet; Naturund Geisteswissenschaftler, Künstler und Fachleute trugen zum Ruf der deutschen Nation in der Welt bei. Im 1. Weltkrieg wurden viele Juden für ihren Heroismus mit dem Eisernen Kreuz ausgezeichnet; 12.000 deutsch-jüdische Soldaten fielen an der Front.

Die hier untersuchte Emigration, die nicht die erste jüdisch-deutsche Emigration nach Argentinien darstellt, setzt mit der Machtergreifung Hitlers am 30. 
Januar 1933 und dem Beginn der Judendiskriminierung ein. Als Titel dieser Arbeit haben wir "Geschichten einer Emigration deutscher Juden nach Argentinien (1933-1939)" gewählt, da der letzte Befragte sein Geburtsland 1939 verließ'. Laut der Encyclopedia Judaica, Band VII, Deutschland, kamen in diesem Zeitraum 25.000 deutschsprachige Juden nach Argentinien. Diese Zahl beinhaltet die Flüchtlinge aus den besetzten Gebieten (s. Tab. 1). Am 31. Oktober 1941 untersagte Deutschland die Auswanderung von Juden, und am 25. November 1941 wurde allen, die emigriert waren, die deutsche Staatsbürgerschaft entzogen.

\section{Tabelle 1}

\section{Ausreise aus Deutschland}

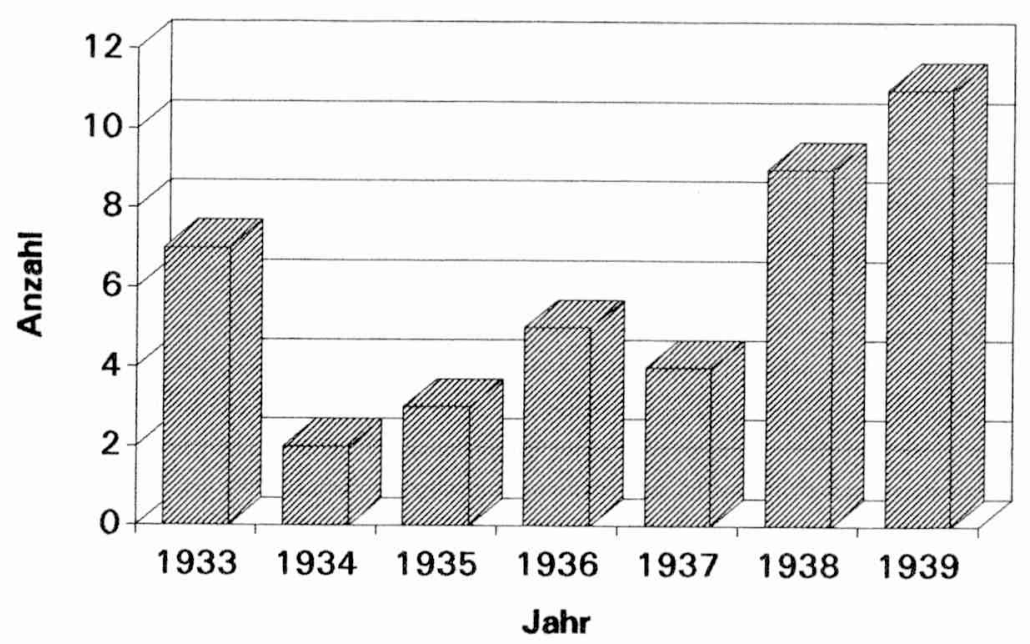

Grundsätzlich zeigt die deutsch-jüdische Einwanderung zwischen 1933 und 1939 andere Züge als die spanische und italienische, was sich auch auf einen Großteil der polnisch-jüdischen und russisch-jüdischen Immigration vor 1933 ausweiten läßt: Sie gingen auf die chronische sozioökonomische Situation von Hunger und Armut in diesen Ländern zurück und wurden von Bevölkerungsgruppen getragen, deren wirtschaftliche Verhältnisse extrem schlecht waren. Unter den deutsch-jüdischen Immigranten gab es dagegen weder Armut noch dem Kleinbürgertum und der gesellschaftlichen Mittelschicht an; es waren keine Landwirte. Diese Tatsache erklärt, daß über $40 \%$ der Flüchtlinge vor allem nach Buenos Aires gingen, $60 \%$ sich in

${ }^{1}$ Die Studie ist 1991 erschienen (s. Levin 1991). Die Untersuchung wurde von der Bundesrepublik Deutschland und die Publikation durch das Goethe-Institut München gefördert. 
der näheren Umgebung der Hauptstadt und $5 \%$ im Landesinnern niederließen, was dem Muster der Bevölkerungsverteilung in Deutschland entspricht. Dort lebte während des untersuchten Zeitraums die überwiegende Mehrzahl der jüdischen Bevölkerung in urbanen Zentren (s. Tab. 2).

\section{Tabelle 2}

Jetziger und früherer Wohnsitz

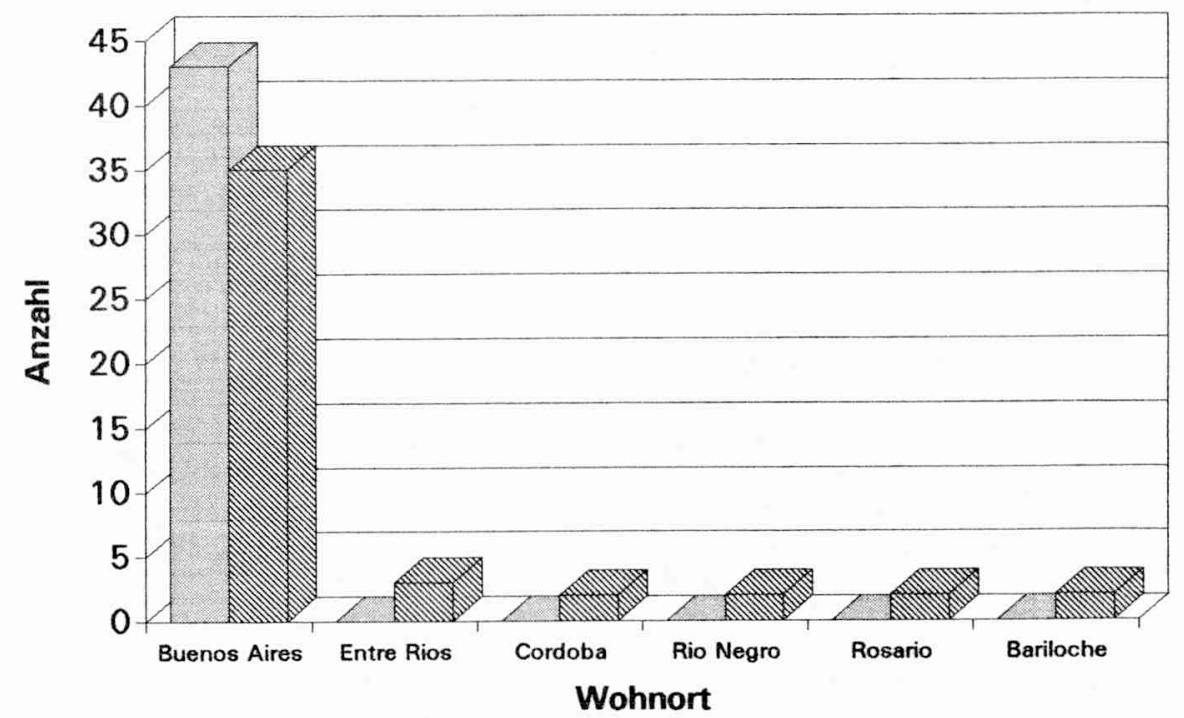

Jetziger Wohnsitz $\mathbb{N}$ Früherer Wohnsitz

Sie waren nicht arm, aber staatenlos; einige besaßen weder $\mathrm{Pa}$ noch Visum. Viele kamen illegal ins Land, mit gefälschten oder gar keinen Papieren. Einige hatten Familie, andere nicht. Sie wußten nichts über Argentinien. Sie hielten an ihrer Sprache, ihren Gebräuchen, ihrer Lebensweise fest, was zu einer ganz charakteristischen Endogamie führte.

Bei diesen Einwanderern, die weder Armut noch Hunger kannten, kann man von einer guten Integration in den Arbeitsmarkt sprechen. Dies belegt eine Studie, die 50 Jahre nach der letzten Einwanderung unter Befragten im Alter zwischen 51 und 88 Jahren gemacht wurde, von denen einige noch auf den Armen ihrer Mütter nach Argentinien gekommen waren. Allgemein fanden Frauen schneller Arbeit, da sie als Kindermädchen, Gouvernanten oder in der Bekleidungsindustrie angestellt wurden. Die Frauen in höherqualifizierten Berufen konnten manchmal sogar ohne 
eine Anerkennung ihrer Titel ihre Tätigkeit wieder ausüben. Für die Männer in gehobenen Positionen dagegen stellte die Anerkennung der deutschen Titel ein Hindernis für den erneuten Einstieg ins Berufsleben dar. Einige betrieben diese Anerkennung der Titel, andere arbeiteten als Angestellte großer Unternehmen oder machten sich als kleine Handelsunternehmer selbständig. Ein extrem hoher Prozentsatz verdiente sich seinen Lebensunterhalt im kulturell-künstlerischen Bereich: Opernsänger, die Karriere machten, Musikvirtuosen und Berufsmusiker in Orchestern, Film-, Musik- und Theaterkritiker (s. Tab. 3).

\section{Tabelle 3}

\section{Beschäftigung in Argentinien}

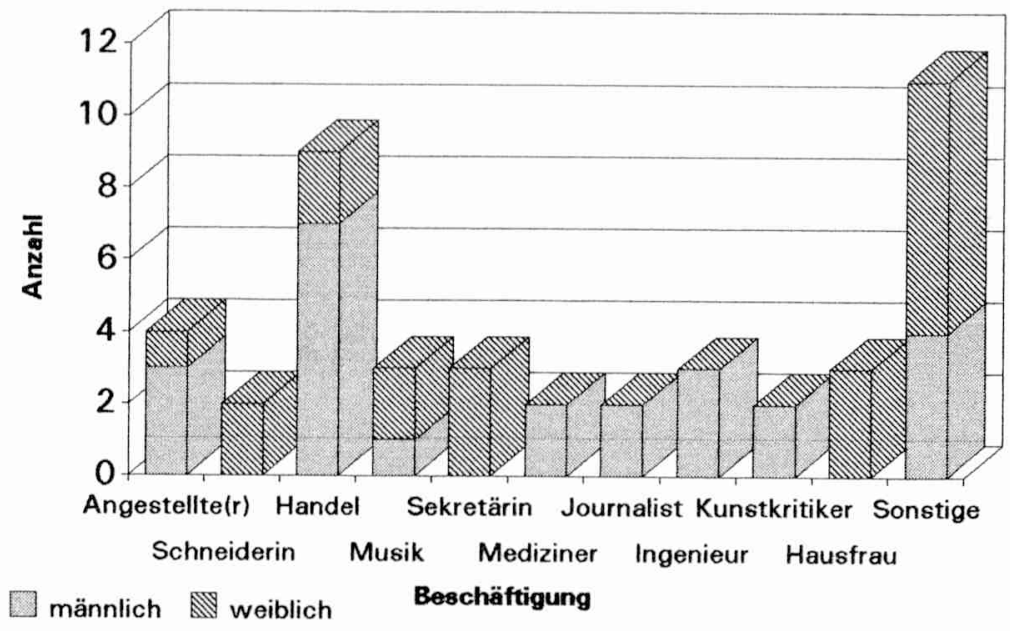

männl. weibl.

$\begin{array}{lrr}\text { Angestellte(r) } & 3 & 1 \\ \text { Schneiderin } & & 2 \\ \text { Handel } & 6,8 & 2 \\ \text { Musik } & 1 & 2 \\ \text { Sekretärin } & & 3 \\ \text { Mediziner } & 2 & \\ \text { Journalist } & 2 & \\ \text { Ingenieur } & 3 & \\ \text { Kunstkritiker } & 2 & \\ \text { Hausfrau } & & 3 \\ \text { Sonstige } & 4 & 7\end{array}$

"Sonstige Beschäftigung"

1 Zahnarzt 1 Bewegungstherapeut

1 Rabbiner 1 Sportlehrerin

1 Unternehmer 1 Lehrerin

1 Anwalt 1 bildender Künstler

1 Buchhändlerin 1 Sänger 
Im allgemeinen gründeten die Befragten Kleinfamilien mit wenigen Kindern, was typisch für das europäische Bürgertum war, und Scheidungen kamen praktisch nicht vor. Daraus läßt sich schließen, daß der Schock der durch die Verfolgung verursachten Immigration bei der Schließung dieser Ehen insofern eine Rolle spielte, als sie angesichts der Vorstellung neuer Verfolgungen und Vertreibungen entschieden, nur wenige Kinder zu haben, um eine erneute Emigration zu erleichtern. Dies ist allerdings nicht die einzige Erklärung. Betrachtet man die Sozialstruktur Argentiniens, innerhalb derer sie sich etablierten, so stellt man fest, daß die typische argentinische Familie zwei oder drei Kinder hatte, was die Immigranten sich neben vielen anderen Dingen aus ihrem kulturellen Umfeld zum Vorbild nahmen (s. Tab. 4). Das Schul- und Ausbildungsniveau der Kinder dieser Immigranten ist sehr hoch: In der Regel besuchten sie weiterführende Schulen oder Universitäten (s. Tab. 5). Sehr viele Personen lernten den Ehepartner in Argentinien kennen, heirateten dort ebenfalls deutsche Juden, und nur ein verschwindend geringer Prozentsatz ließ sich später scheiden. Das heißt, die Endogamie, oder das Verbleiben im eigenem Milieu, in ihrem "Ambiente", schuf stabile Beziehungen. Die Befragten selbst gaben an, sich gegenseitig besonders zu brauchen, weil sie eben in einem fremden Land lebten.

\section{Tabelle 4}

\section{Kinderzahl}

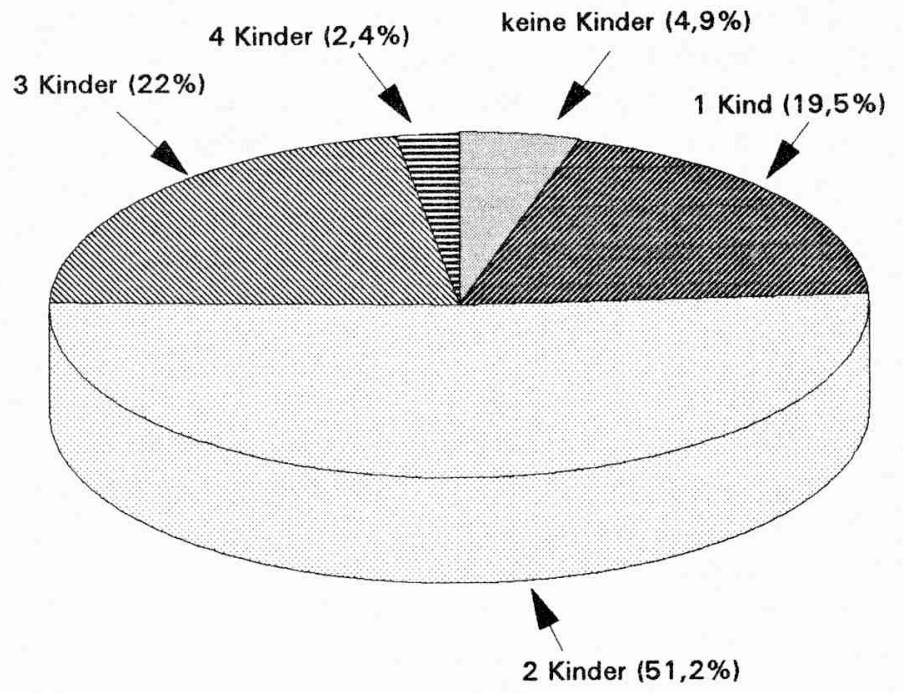




\section{Tabelle 5}

\section{Schulbildung der Kinder}

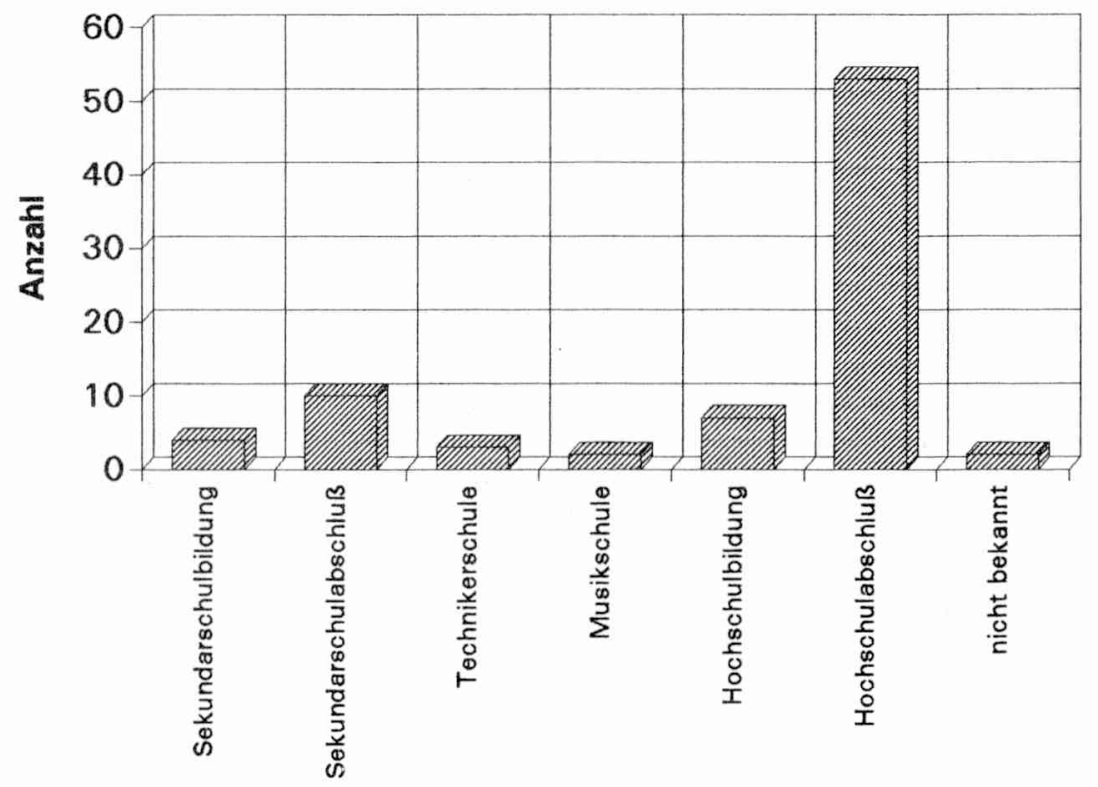

Schulart

Die deutschen Juden leisteten ihren Beitrag zur argentinischen Gesellschaft in besonderer Weise: Sie nutzten die Gelegenheiten, die sich gerade boten, doch ihr Interesse war auf Europa gerichtet, weshalb sie auch nur marginal am argentinischen Leben teilnahmen. Sie besuchten Konzerte und Ausstellungen, waren in ihren Gemeinden präsent und lasen viele Bücher, aber sie waren politisch wenig engagiert, und neben ihren kulturellen und religiösen Aktivitäten beteiligten sie sich kaum am sonstigen gemeinschaftlichem Leben. Möglicherweise geht diese apolitische Haltung auf die Angst zurück, die eine politische Betätigung bei diesen Immigranten hervorrufen mußte, die Opfer eben einer Politik waren, deren Inhalte sie nicht verstanden, die aber ihr Zuhause und ihr Leben zerstört hatte. Dies gilt allerdings nicht für die Generation der heute 50jährigen, die ihre ganze Kindheit und Jugend in Argentinien verbracht haben.

Im Gegensatz zur italienischen und spanischen Immigration, wo $50 \%$ der Betroffenen wieder in ihr Heimatland zurückkehrten, wie Untersuchungen anerkannter Soziologen zeigen, ist die jüdisch-deutsche Einwanderung eine Immigration ohne Wiederkehr.

Von den befragten Personen hatten alle Deutschland nach dem Krieg wieder besucht (s. Tab. 6). Dies ist recht vielsagend, da die Umfragen ihre eindeutige Ablehnung zeigen, in ihr Geburtsland zurückzukehren und da die Besuche dennoch darauf schließen lassen, daß auch Gründe für eine Rückkehr sprechen 
(z.B. Wiedergutmachungsbemühungen). So würde auch erklärbar, daß berufliche (wissenschaftliche Kongresse) und geschäftliche Gründe (unternehmerische Fortbildungskurse, Schulungen in deutschen Zweigniederlassungen) sowie Urlaubsreisen zum Vorwand für einen Deutschlandbesuch genommen wurden und man die von der Geburtsstadt ausgesprochenen Einladungen annahm, die oft schon ganz sehnsüchtig erwartet worden waren. Wie die Befragungen ergaben, besuchten viele der Emigranten die Friedhöfe, andere suchten mit gemischten Gefühlen ihr Geburtshaus auf, gingen durch "ihre" Stadt oder "ihr" Dorf. Alle die solchen Einladungen folgten, wußten diese und die damit verbundenen Aufmerksamkeiten zu schätzen (Empfang durch den Bürgermeister, Treffen mit Gruppen, die sie willkommen hießen). Bei den Zusammentreffen mit ehemaligen Bekannten und oft auch Altersgenossen bekamen diese Menschen wieder ein Gesicht (das reale Gesicht der Vergangenheit), einen Namen und einen Vornamen. Sie waren nicht länger die namen- und gesichtslosen Gespenster, die, obschon sie die gleiche Sprache sprachen, in der Vergangenheit so großes Entsetzen verbreitet hatten.

\section{Tabelle 6}

\section{Erster Besuch in Deutschland}

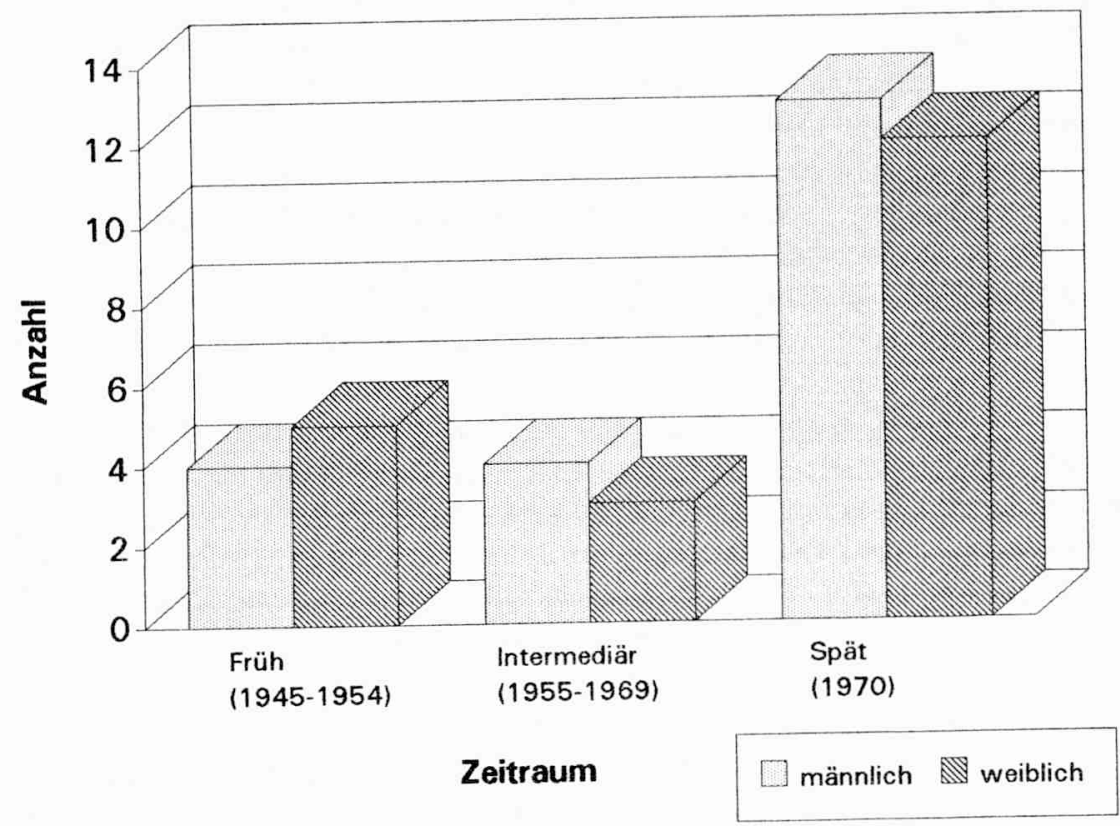


Der Einladung durch die Geburtsstadt kam in ihren Funktionen als Wiedergutmachung eine besondere Bedeutung zu: Dem Gedanken: "Die Leute, die mich aus dem Land getrieben haben, laden mich jetzt ein und nehmen mich wieder auf". Darüber hinaus gab dies Gelegenheit zu erfahren, was mit den damals Verschwundenen geschehen war

Auf der anderen Seite fühlten sich viele bei Besuch wieder zu Hause, was sie in einen großen Gewissenskonflikt stürzte: Es war äußerst schmerzhaft zu erfahren, wie tief diese deutschen Wurzeln reichten und wie authentisch sie waren. Viele der Befragten warteten sehnsüchtig auf Bücher aus ihrer Heimatstadt, um zu sehen, ob ihre Verwandten und deren spätere Schicksale erwähnt waren: Deportation, natürlicher Tod, Selbstmord (zwischen 1941 und 1944 war in Berlin die Selbstmordrate älterer Menschen sehr hoch, da sich viele lieber vergifteten, als ihre Heimat zu verlassen).

Viele die überlebten und in Argentinien Familie, Haus, Arbeit und finanziellen Wohlstand fanden, entwickelten ein Schuldgefühl gegenüber ihren Angehörigen, die in Konzentrationslagern umgekommen waren. In diesen Fällen wurde die Tatsache, sich gerettet zu haben, als tadelnswert erlebt, als Schuld, der sie sich nicht stellen konnten.

Aus der Studie geht hervor, daß alle Befragten "das Deutsche" lieben und überbewerten. Obschon ein Teil der Personen eine deutliche Empörung angesichts der unter dem Nationalsozialismus geschehenen Ereignisse empfand, tendierten die Befragten dazu, diese Vorfälle vom deutschen Wesen zu trennen, wie einen religiösen Glauben, der in der persönlichen Geschichte oder der Familientradition verborgen liegt. Der - angepaßte - Deutsche jüdischer Konfession ersetzte den Glauben seiner Vorfahren durch das Konzept Vaterland, das er mit Deutschland und seiner Kultur verband und denen er sich voller Stolz, und zu seinem eigenen Nutzen, anpaßte. Aber der Glaube der Deutschen, diese "Religion", heißt Patriotismus (wie Friedrich Dürrenmatt in einem Essay über den Patriotismus zu verstehen gibt) und der schloß die Juden nicht mit ein, sondern grenzte sie im Gegenteil explizit aus, verfolgte sie und schrieb ihnen die Schuld an allen Übeln zu, unter denen das 'deutsche Vaterland' litt.

Die Deutschen so zu sehen, wie sie sind, ist für die Befragten zum heutigen Zeitpunkt zu schwierig. Statt dessen ist es zu einer Trennung und Distanzierung von allem Negativen, von Gewalt, Unterwerfung, Aggression gekommen. Es besteht nur fort, was gut und geschätzt ist: Eigenschaften wie Ordnungssinn, Genauigkeit und Korrektheit. Man läßt sich von Romantik, Musik und Poesie über die Maßen begeistern. Die deutschen Juden in Argentinien schufen sich zu Hause, zwischen Bildern, Büchern und Pflanzen, Möbeln und Porzellan, Kaffee und Kuchen, einen Teil "ihrer deutschen Heimat" neu. Viele der deutschen Juden in Argentinien sprachen hier und mit ihren Kindern weiterhin Deutsch; möglicherweise verhinderte dies in gewisser Weise die Desintegration, das Auseinanderbrechen von Strukturen; es war die Negation der Vertreibung aus Deutschland: Sie bewahrten sich ihr Deutschland. Auch wenn dies die eigene 
Assimilation und die der Kinder verhinderte, bewahrte es ihnen ihr Bewußtsein. In Argentinien fanden die deutschen Juden eine, oft abgelehnte, Nationalität, aber keine Identität. Die deutschen Juden konnten und wollten sich nicht mit Argentinien identifizieren, sie verschafften sich eine neue Persönlichkeit, behielten aber die alte bei.

Das Gefühl, Deutscher zu sein, das über Generationen von in Deutschland geborenen Familienangehörigen vermittelt wurde, ihre Sprache, die kulturellen Aktivitäten und die berufliche Betätigung sowie die Zwangsimmigration riefen bei fast allen befragten Personen eine verwirrende Gefühlswelt hervor. Diese widersprüchlichen Gefühle sind symptomatisch für die deutschen Juden und unterscheiden sie damit von dem allgemeinen Schicksal, das den europäischen Juden ab dem 18. Jahrhundert widerfuhr. Bei denjenigen Befragten, die ihren Glauben täglich praktizieren, hat es den Anschein, daß jüdische Religion und Deutschsein voneinander getrennt sind. Doch fast niemals stehen Religion und Nationalität in Konfrontation zueinander. Im Gegenteil, es wird eindeutig erklärt, daß beide einander potenzieren, nicht ausschließen, und daß das Gefühl der Verbundenheit mit der jüdischen Religion in dieser Gruppe auf das engste mit dem Stolz, Deutscher zu sein, verbunden ist. Die Vertreibung aus Nazideutschland ließ dann ein Knäuel der Verwirrung entstehen, in dem sich das jüdische Wesen und das Deutsche sowie später das Argentinische eng ineinander verwoben - wie soeben erklärt - , was eine klärende Abgrenzung aller drei Bewußtseinsstadien voneinander verhinderte.

Die Symbiose mit der deutschen Eigenart wird vom jüdischen Standpunkt aus in der Tatsache deutlich, daß es am höchsten bewertet wurde, deutscher Staatsbürger jüdischen Glaubens zu werden, der alle germanischen Attribute des Deutschlands der Vornazizeit aufweisen sollte. Vielleicht stellte deshalb die große Liebe zum deutschen Wesen die Möglichkeit dar, sich für einen Moment als Mitteilhaber an den Geschicken des Landes zu fühlen. Das "Deutsche" erlaubte es dem Juden, sich zu entfalten und zu wachsen. Als die deutschen Juden durch den Entzug der deutschen Staatsbürgerschaft, des höchsten Gutes, das diese Gruppe besaß, den Rückhalt des Staates verloren, waren sie überrascht, entsetzt und wie gelähmt. Die enorme Enttäuschung, die durch den Verlust der staatlichen Unterstützung, den Entzug der deutschen Staatsbürgerschaft hervorgerufen wurde, ist vergleichbar mit dem Verlust und der Ablehnung des Vaters, der seine eigenen Kinder nicht mehr als solche anerkennt. Der Staat, in Stellvertretung des Vaters, verstieß jene, die sich für seine Kinder hielten, er bezeichnete sie als Fremde und vertrieb sie des Landes. Die Emigranten fanden sich in jüdisch-deutschen Institutionen zusammen, obschon viele von ihnen in Deutschland dem Judentum nicht sehr nahe standen: Nachdem sie das StaatlichDeutsche verloren haben, blieb noch das Idiomatisch-Deutsche, und das Jüdische rückte als Bindeelement für diese Einwanderergruppe in den Vordergrund.

Die Bedeutung der Sprache als Paradigma der deutschen Kultur ist bereits analysiert worden. Die Sprache, der Klang, die Musik bilden eine Art klanglicher Umhüllung, die das Kind nährt und es wachsen, sich entwickeln läßt, 
ähnlich wie es im Mutterleib von Fruchtwasser umgeben ist. Die deutsche Sprache, die Musik, die sie in ihrer Kindheit hörten, bildeten für die Emigranten, als sie in Argentinien blieben, eine Art Schutzhülle, die den Verlust der Heimat weniger schmerzhaft machte. Man konnte ihnen alles nehmen, Staatsbürgerschaft, Ländereien, ihr Hab und Gut... doch trugen sie in sich den Klang ihrer Muttersprache. ${ }^{2}$

Aus dem Spanischen von Maria Maier

\section{Bibliographie}

Aus Politik und Zeitgeschichte. 1988. Beilage zur Wochenzeitung Das Parlament/Bonn. 21. Oktober. Bonn, B43/88.

Bracher, Karl D.; Manfred Funke; Hans-Adolf Jacobsen (Hg.) 1987. Die Weimarer Republik 1918-1933. Düsseldorf: Droste.

Enciclopedia Judaica. 1971. Jerusalem: Keter Publishers.

Galliner, Nicola. 1987. Wegweiser durch das jüdische Berlin. Berlin: Nicolai.

Gilbert, Martin. 1988. Atlas de la Historia Judía: 121 mapas comentados. Buenos Aires.

Kultur Chronik. 1989. Noticias e informaciones de la República Federal de Alemania. Bonn: Inter Nationes, Nr. 5.

Levin, Elena. 1991. Historias de una emigración (1933-1939). Alemanes judios en la Argentina. Buenos Aires: Manrique Zago.

Melcher, Peter. 1986. Weissensee: ein Friedhof als Spiegelbild jüdischer Geschichte in Berlin. Berlin: Haude \& Spener.

Michman, Dan; Yehuda Bauer; Judy Baumel; Yechiam Weitz. 1986. "El Holocausto", un estudio histórico. Israel: Universidad Abierta.

Schrader, Achim. 1989. "Desaparecidos sin dejar huella? La República Federal de Alemania y los emigrantes judío-alemanes en la América Latina". In: Estudios Migratorios Latinoamericanos (Buenos Aires), 11.

Sitton, Thad, et al. 1984. Historia oral. Una guía para profesores (y otras personas). México: FCE.

${ }^{2}$ Eine detaillierte Ausführung dieser Untersuchung, die von der Bundesrepublik Deutschland gefördert wurde, ist erschienen in: Elena Levin. 1991, dessen Publikation dank der Unterstützung des Goethe-Instituts München möglich geworden ist. 